\title{
Use of graphical modality in a collaborative design distant setting
}

\author{
Stéphane Safin ${ }^{\mathrm{a}^{*}}$, Roland Juchmes ${ }^{\mathrm{a}}$, Pierre Leclercq ${ }^{\mathrm{a}}$ \\ ${ }^{\mathrm{a}}$ LUCID - Lab for User Cognition and Innovative Design, University of Liège, 1 chemin des chavreuils B52, 4000 \\ Liège, Belgium
}

\begin{abstract}
In this paper, we present an original environment, the Distributed Collaborative Design Studio (DCDS) which allows multimodal real-time remote exchanges, and which aims at re-creating remotely the conditions of co-present meetings. This environment associates a videoconference tool (supporting verbal and non-verbal communication) and an original realtime shared digital hand-drawn sketches system (supporting graphical communication). The users can import documents and annotate them in real-time at distance. In this paper, we are interested in studying the use of the graphical modality (digital sketch and document annotations) as a tool for collective design and remote communication. This study takes place in the framework of a collaborative architectural design studio, which has taken place during 3 month, and gathering students from Belgium and France to work together in 3 small groups mainly at distance. The study focuses on the role of graphical modality in the synchronous remote meetings supported by the DCDS. We identify the types of digital annotations made on the imported document, thanks to the electronic pen, and their role in the cognitive processes of designing and in the collaboration and communication processes. We also identify the different practices of digital sketching, according to the group and its collaborative strategies. We discuss the utility of the graphical modality as an efficient support for collaborative synchronous activities and show that the DCDS environment supports different strategies of collaborative design (co-design and distributed design). We conclude on recommendations for improving the system and for designing sketch-based collaborative environments.
\end{abstract}

Keywords: CSCL; architecture; multimodal collaboration; pen-based interaction

\section{Introduction}

In a wide range of activity sectors, collaboration has been intensified, notably in the design domains. Collective work is increasingly organized simultaneously (rather than sequentially as it used to be in the past). Moreover, design teams are often geographically distributed, and the need for distant realtime interaction is consequently emerging.

While the best way of ensuring effective coordination and collaboration remains face-to-face meetings, convening all participants at the same time in the same place can often be problematic. Regular meetings, which are by their nature difficult to convene spontaneously, do allow for collective reflection to take place and such meetings remain the most ef- fective way of turning complex projects into reality. However, when the meetings adjourn, each participant follows up on their own part of the work, with the risk of falling out of synch with other participants before the next meeting. Moreover, the meetings are conditional upon copresence, with all its associated travel costs and, above all, the lengthy immobilization of human resources. During such meetings, not everyone has every member of their team available or all their documentation, equipment and usual working "tools" to hand. This can hinder the input they can make to the meeting.

Synchronous and asynchronous working procedures between participants who are geographically separate have naturally developed in order to overcome the difficulties associated with organizing co-

\footnotetext{
*Corresponding author. Email: stephane.safin@ulg.ac.be
} 
present meetings, supported by today's widely available technology. Current communication technology, such as telephone, email, file servers and videoconferencing help in this process of coordination. But these remain limited in terms of collaborative working, particularly when it comes to creative or problem-solving tasks. Such tasks always develop synchronously, using diagrams and notes shared around the same table. Although the available means of communication do greatly facilitate the exchange of information, they turn out to be very limited when compared to the possibilities of actual copresent meetings.

Indeed, face-to-face communication possesses a certain number of important features such as temporal pattern (synchronous), completeness of the channel (verbal, visual, gestural, graphical...) and implicit communication. The available technology cannot support all of these features and remains limited. An analysis of collaborative working situations suggests that this limitation is mainly due to a crucial failure of shared synchronous representation.

File-sharing technology such as emails, file servers or electronic document management systems, for example, function in an asynchronous mode. This forces collaborators to construct information incrementally, by successively accumulating content ('versioning'), rather than incorporating it. The decision-making process thus rapidly becomes less consensual. Moreover, making decisions through successive interventions does not encourage connections to be made between different points of view or the incorporation of all opinions into the final decision.

Furthermore, real-time communication tools are not designed to convey representations of content but only comments on them, either by voice or videoconferencing. Thus, telephone, video-conferencing or web chatting do not allow for an evolving graphic representation to be shared.

This difference between modalities of exchange is greatly disruptive to the efficacy of orchestrated collaborative working, which continues to favour copresent meetings.

Finally, it is important to note that all complex activities are characterized by the use of numerous documents, which are annotated or modified. In architecture, these documents are an integral part of the design process, translating existing perceptions and representations, then simulating and testing possible interventions: these documents include sketches, drafts, plans, specifications, etc. They are jointly produced by multi-disciplinary teams and are modified in real time (through annotation) to support the collective decision-making process.

Nevertheless, some devices and environments are emerging, allowing rich distant interactions, and are increasingly used in professional but also in educational settings. In this paper, we present an original environment, the Distributed Collaborative Design Studio (DCDS) which allows multimodal real-time remote exchanges, and which aims at re-creating remotely the conditions of co-present meetings. This environment associates a videoconference tool (supporting verbal and non-verbal communication) and a real-time shared digital hand-drawn sketches system (supporting graphical communication). The users can import documents such as plans, images and models, and annotate them in real-time at distance.

In this paper, we are interested in studying the use of the graphical modality (digital sketch) as a tool for collective design and remote communication. This study takes place in the framework of a collaborative architectural design studio, which has taken place during 3 month, and gathering students from Belgium and France to work together in 3 small groups at distance. The students are supplied with asynchronous means of collaboration (dedicated database, mails...) and our synchronous multimodal collaborative environment, the DCDS.

\section{Collaborative design}

Collaborative design requires three classes of activities: task-oriented activities, process-oriented activities and interaction management activities [7].

Task-oriented activities are directly related to the content of the design. Usually, one can distinguish problem framing, solutions generation and solutions evaluations. Those activities occur in individual design, but also in collective design, through argumentation processes [5]. Communication is therefore an essential point for solving the design problem: a common understanding of the problem allows to structure it (e.g. suggesting goals), the ideas must be generated by the different persons and communicated to the group (e.g. propositions of design) and, to be efficient, the ideas must be collectively evaluated through communication processes (e.g. criticisms). Stempfle \& Badke-Schaub [16] showed that those content-oriented activities account for about $2 / 3$ of the interactions between group members. 
Process-oriented activities are necessary to coordinate group actions. These activities are linked to the management of viewpoints, the synchronization and coordination, the conflict management, the building of a common knowledge [17]. Two modes of coordination usually can take place in collaborative design [6].

- Distributed design where the actors perform distinct but interrelated tasks, each one mobilizing its own resources and its own temporality to carry out specific objectives serving the joint project. The actions are simultaneous, but not joint. The key issue is the coordination of different partners' activities and their temporal articulation.

- Co-design where all the designers respond to the problem in an integrated way, share common goals, generate solutions and evaluate them together. The challenge of this type of collaborative design is the cognitive synchronization, i.e. the creation, through actions of communication, of a shared common context that allows the entire group to coordinate more effective action.

In collaborative activities, primarily in distributed design activities taking place at a distance, the management of interdependencies between tasks and designers, actors need as to have a collective consciousness of the situation, of the changes made on the design object, of the tasks, and of the partners' skills and activities. This mutual consciousness is called situation awareness [3]. While in face-to-face situations actors share a common context, remote interaction can be disrupted by many constraints: reduction of the richness of communication channels (e.g. reduced field of view) and difficulty in sharing information and objects (e.g. troubles communicating spatial reference). To achieve this mutual awareness, it is necessary to share a part of the context.

The different views on the object must be coordinated and integrated to build a common vision. This mechanism, called grounding, involves the construction of a common reference space consisting of all the knowledge that group members have in common and are aware that they have in common. This space is called a shared common ground [4] or joint problem space [10]. This common ground is not just understanding who undertakes any action and how the task is conducted globally, but rather building a strong inter-understanding to understand each other and solve the problem together. The grounding affects both the problem (a framework for the generation and evaluation) but also the procedures and representations and the knowledge that the partners of each other. The negotiation of common ground is a prerequisite for negotiating solutions.

Interaction management activities. These include all activities related to the process of communication. To communicate, one need to develop a message, but also to verify that this message has been understood. It is also necessary to provide clues and positive evidence to the partner(s) to show that the message has been understood: acquiescence, confirmations, start of next speaking turn, and so on [4]. These activities are simple in the case of face-to-face communication using everyday language, but are much more complicated in the case of remote communication, of asynchronous exchanges and in the case of high degree of sophistication or abstraction messages.

The communication has therefore a "cost". The different costs of communication are associated with the constraints of environments for sharing and supporting collaboration. The media of communication can be characterized by several properties that can facilitate exchanges and the construction of a shared common referent: possibility to see and hear each other, simultaneity of actions, sequentiality of the messages, reviewability, etc. [4].

\section{DCDS}

In order to support remote synchronous collaboration, the LUCID-ULg lab has developed the Digital Collaborative Design Studio (DCDS)

This prototype is composed of two parts. A hardware part, the Virtual Design Desktop (figure 1), which consists of an electronic A0 drawing table with a suspended ceiling equipped with a projection system offering a large working surface (approximately $150 \times 70 \mathrm{~cm})$. An electronic pen allows the drawing of virtual sketches onto this surface. Manipulation widgets are especially designed to interact only with the stylus in this environment.

A software part called SketSha (for sketch sharing - figure 2) is a shared drawing environment allowing several users to be connected to the same virtual drawing space. Various functionalities, such as a panel of coloured pens (and an eraser) and a navigation tool (to zoom, translate, rotate), are available through intuitive graphical widgets. Some layout facilities have also been included in the prototype, such as the possibility of drawing and managing different sheets of virtual paper, of deleting or duplicating them, and of managing their transparency. The soft- 
ware also allows users to import Computer Assisted Design (CAD) plans and bitmap images. This software captures the strokes that compose the sketch, shares them between the different distant locations (through a standard internet connection) and transmits the complete information in real time onto the active boards through video-data projectors.

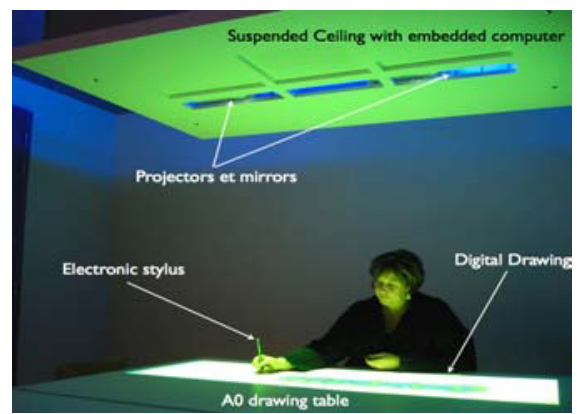

Fig 1 : Virtual Desktop.

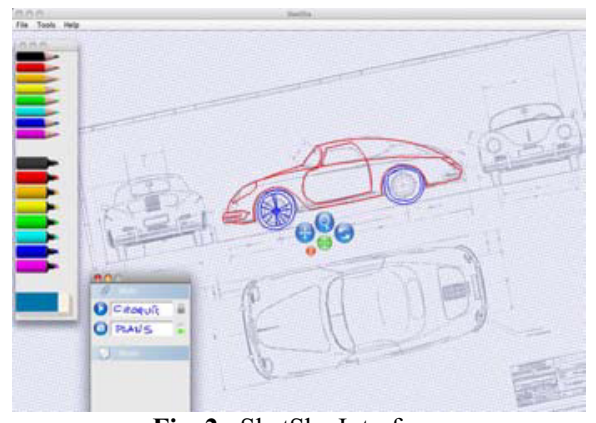

Fig. 2 : SketSha Interface.

The system is completed by a 24 -inch screen with an integrated camera, which allows the participants to see and talk to each other, on an almost 1:1 scale, during a real-time conference. Pointing, annotating and drawing are possible due to the electronic pen. Social exchanges are transmitted through the external modules of video-conferencing in order to support the vocal, the visual and the gestural aspects of the collaboration.

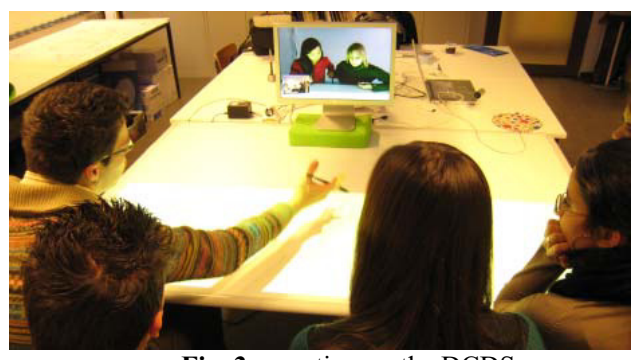

Fig. 3 : meeting on the DCDS
Following the user-centred framework underlying the development of the DCDS, the system has already been tested in different short and long collaborative work settings with students and professionals, see $[1,2,8,9,13,14]$

\section{Issues}

The question raised in this paper are the following.

"Does the graphical modality supplies people with means to manage a coupled collaboration ?"

As a way to enrich collaboration, by supplying the designers with a mean to recreate copresent meetings at distance, we expect that the adjunction of the graphical modality will support more coupled versions of collaboration. We also expect that this modality will encourage more collaboration among group members.

\section{"How the graphical modality is used to support the collaborative design process?" \\ We wish to understand how the designers use the graphical modality to support their design process (generating solutions, reframing the problem and evaluating solutions) and their collaborative process (awareness, grounding, interaction management).}

To answer these questions, we followed the activity of three groups of student during a 3-months architectural design workshop, analyzed their collaborative process and observed their uses of the graphical modality during weekly collaborative design sessions supported by the DCDS.

\section{Setting}

This study has taken place in the framework of a collaboration between the Nancy School of Architecture (France) and the Faculty of Applied Sciences of the University of Liège (Belgium).

3 groups of 5 students (mixed with French and Belgian students) worked during one term (3 months) on an architecture program. Each group had to design collaboratively and remotely a building (a cultural center), from a program completely defined.

For this purpose, the groups had several tools at their disposal :

- Asynchronous collaborative tools (mails, file exchange servers,...)

- Synchronous collaborative tools (chat, videoconferencing...) 
- A one-hour meeting each week on the DCDS. They were allowed to bring their documents in the system, annotate it during their discussion, save the edited documents.

This study focus on the weekly synchronous meetings with the DCDS. We videotaped each session and recorded all the documents, digital drawings and digital annotations ${ }^{1}$. The video data represent 8 one-hour meetings for each of the 3 groups, for a total of 24 hours of video.

\section{Method}

Our method is composed of three parts : an analysis of the collaborative process, a content analysis of the graphical traces, and an activity analysis of the drawing activity.

\section{Assessment of the collaboration}

In order to understand and describe the collaborative process, we used three methods.

- The direct observation of the group during their distant synchronous meetings.

- A presentation of a reflective analysis made by the groups on their own collaborative process (mandatory for the workshop).

- A video analysis supported by a grid to assess the quality of collaboration. This grid, inspired by Spada's works in the CSCL domain (see [15]), allows to quickly assess the collaborative process according to seven dimensions : fluidity of collaboration, sustaining mutual understanding, information exchanges for problem solving, argumentation and reaching consensus, task and time management, cooperative orientation, and individual task orientation. These dimension cover the task-related, group management and communication processes. As this tool is not the main focus of the paper, we do not describe it here deeply. We use it in our study to have a global score on the collaboration quality. The interested reader may find more information $[1,2]$.

These three methods allow us to describe and compare the modes of collaboration of the three

\footnotetext{
${ }^{1}$ In this paper, we refer as "document" any file imported on the system, "annotations" all the graphical traces drawn on the documents with electronic pen in the DCDS and "sketches" the graphical traces drawn on a white virtual paper (i.e. not on a document).
}

groups. Based on this description, we can compare the collaborative sketching behaviors of the groups.

\section{Graphical traces analysis.}

We recorded all digital drawings during the sessions and all annotations on imported documents. We distinguish 4 types of annotation, depending on whether they have a strong or weak geometrical dependency with the underlying document, and whether they bring new information to the document ${ }^{2}$.

- Pointing. In this type of annotations, one or more simple elements of the document are emphasized, but no information is added. This emphasis may be made by a circle surrounding a portion of the drawing or by an arrow for example. There are no strict correspondence between graphic annotation and the annotated document.

- Highlighting. These are graphic annotations precisely superimposed to the original document, but bringing no new information. These annotations are redundant with the images they complement and highlight certain elements of the drawing or set of decisions.

- Elements. Here, simple graphical elements (arrows, construction lines, etc.) add some information to the original image, but are not in close geometrical relation to it. These elements complete the underlying document but they do not modify it.

- Drawings. These are sketches superimposed on the document. These drawings are figurative and aim to modify the document. They are acts of designing.

\section{Activity analysis}

Finally, we made video analysis of a sample of video extracts in each group. These video have been analyzed by an ergonomist and an architect together. This sample has been made in order to have a representation of all kinds of drawings and annotation, in each group and in different moments in the design process.

We tried to identify the uses of the graphical modality, i.e. digital drawings and annotations, in the collaborative design process. Our observation focus on the roles and functions of annotation in the communicative process, the graphical characteristics of the

\footnotetext{
${ }^{2}$ There should be a fifth category: textual annotations, but we did not observe writings in the shared DCDS space.
} 
drawings and the difference between the groups, according to their modes of collaboration.

\section{Comparison of the 3 groups' collaborative activity}

Clearly, the three groups do not collaborate in the same manner.

The group 1 sets up a "distributed design" process : the members work individually asynchronously, take some decisions, and the propositions are presented to the group in the synchronous sessions. Specific issues are resolved collectively in those synchronous meetings and the work is divided. Each member ends the session with specific tasks to be done for the next weekly synchronous meetings. Between two DCDS sessions, students exchange documents and questions by email. The group is characterized by intermediary scores on the collaboration quality scale during their synchronous meetings. But the group globally manage well the design process : the results of the design is judged as excellent by the teachers.

Group 2 establishes a "co-design" process : key structural decisions are taken during DCDS sessions, a lot of collective propositions are done during those meetings. Between two sessions, students work locally in small groups to implement the decisions and proposition (by drawing plans and model). The formal exchange for explanation and presentation of the documents take place by email before the synchronous sessions, to free up time in DCDS sessions for collective decision making. The group has excellent scores on the quality of collaboration scale all along the process. They are far better than the two other in the dimension linked to argumentation, decision and consensus and the one linked to the balance of the contributions of the group members. They are truly engaged in a collaborative design, where all members participate to all decisions. Their design outcome is judged as excellent by the teachers.

Group 3 is characterized by the establishment of a "distributed design" process and the emergence of conflict early in the workshop. Each student in this group makes his own propositions alone and the DCDS sessions are used to present each own work, to try to convince the others of each own ideas and to require teachers help and comments if necessary. Many asynchronous exchanges are intended to argue and make decisions without real success. The group obtains weak score on collaboration scale. In particular, they are globally weaker than the other groups in three dimensions : fluidity of collaboration, mutual understanding and information exchanges for problem solving. This mode of work strengthens the conflict. After 8 weeks, the conflict being too important, this mode of cooperation is interrupted by the teachers, who impose a formal structure for sharing responsibilities and for distributing the tasks. Thanks to this external help, the group manages to finish the workshop decently. Their design outcome is nevertheless judged as weak.

These short descriptions highlight three clearly different modes of collaboration. In the next sessions, we try to link these modes of collaboration to the usages of graphical modality in the synchronous distant meetings.

\section{Graphical traces analysis}

In parallel to the collaborative process, we observed three different patterns of annotations and digital sketches practices in the collaborative synchronous sessions.

The three groups annotated about half the documents they imported in the system (Between $41 \%$ and $50 \%$ ). But the types of annotations are distributed differently across the groups. Table 1 shows the repartition of the different types of annotations.

\begin{tabular}{|l|r|r|r|r|}
\cline { 2 - 5 } \multicolumn{1}{l|}{} & Pointing & Highlighting & Elements & Drawings \\
\hline GR1 & 0,22 & $\mathbf{0 , 4 3}$ & 0,17 & 0,17 \\
\hline GR2 & 0,13 & 0,21 & 0,24 & $\mathbf{0 , 4 2}$ \\
\hline GR3 & $\mathbf{0 , 3 7}$ & 0,22 & 0,23 & 0,18 \\
\hline
\end{tabular}

In group 1, the pointing and highlighting annotations are mainly used. These two types of annotation do not convey new information to the document. This is consistent with the collaborative process of the group: documents are prepared in advance and presented to the group. There is no need to add new information. Nevertheless, the pointing is not the most frequent annotation: this group uses mainly highlighting annotations. This kind of annotations is more than just communicating on the drawing. We have shown, in individual design, the importance of highlighting for taking decisions [12]. 
Group 2 uses annotation to bring new information to the documents : $2 / 3$ of the annotations are elements and drawings. In particular, this group uses drawings annotations, which are mainly used to generate ideas. This characterizes the common generation of solutions and a common decision process. We may note that this group draw also more digital sketches than the other (on virtual white sheet).

Group 3 manly uses pointing annotation. Once again, this observation reflects the collaborative activity of the group. They take few or no decision together and use DCDS synchronous meetings trying to convince each other that their own solution is better. For this purpose, it seems that pointing is the most efficient way of using graphical modality.

\section{Activity analysis}

In addition to the "static" analysis of the graphical traces, we performed a "dynamic" analysis of drawing and annotation behaviors. We identified several functions to annotations and differences in usage by groups and individuals.

\subsection{Functions of annotations}

According to our observations, digital annotations serve different roles during the collective activities.

Draw attention to one element of the design. This function is to support communication by spatially contextualizing the discourse on the documents. It is the deictic role of annotations. This role is carried by pointing annotations but also by some highlighting annotations. These annotations are similar to pointing gestures and can spatially locate a question or comment. This feature of the annotation is temporary: once made, the trace is no longer necessary.

A second function, close to the previous but however different, consists in putting in correspondence elements present in several documents. Thus, the annotations do not only support the speech, but also help to make connections between multiple representations, on a graphics mode. In the illustration below (Figure 4), the designer, explaining its partners the principles underlying its construction, explicitly shows on five drawings where is the "heart of the project." This function is supported by highlighting or pointing annotations. No new information is spe- cifically added on the document, but this mapping can convey a specific message, namely the identification of several drawings of a common concept.

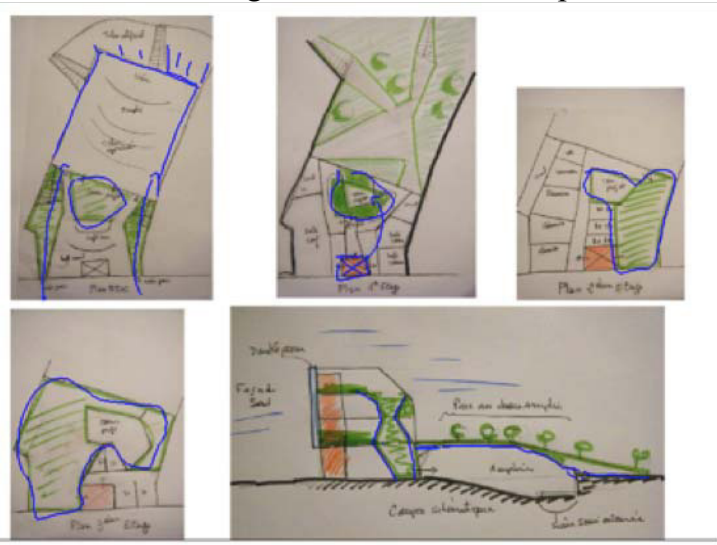

Fig. 4 : identification of the "heart of the project" (in blue) with digital annotations on previous documents (scanned pen-and-paper sketches).

Designers also use annotations to contextualize the document on which they are drawn. They may typically indicate North or elements of surrounding context (road, buildings neighborhood, etc.) and are mainly conveyed by annotation of "elements" type. This contextualization is important in the course of communication, but does not create new information to be conserved. These annotations are explicitly intended to reduce ambiguity and synchronize the different viewpoints.

Another function is to complete the document with information not present explicitly, related to the "functioning", the use or the atmosphere in the building. These are elements that traditional architectural representation does not contain, such as circulation information, ambiance, luminosity, etc. Indeed, plans adopt a strictly geometric perspective on the architectural object. This added information explains how to interpret the plan. The digital annotation, accompanied by speech, can compensate for weaknesses in other representations (models, plans, images, etc.). This additional information will be primarily driven by elements annotations. Some complementary digital sketches can also be made next to the document, such as perspective drawing, detailed design or synthetic cross-section.

Graphical annotations are also used to "synthesize" the document. In this case, the designers highlight specific parts of the plans or models to emphases the main elements of the building. The design is 
synthesized into "functional areas" which make up the premises of the building. This function is performed with highlight annotations and no new information is brought to the document. This synthesis reduces the complexity of the design and some of the uncertainty, but it does not add precision to the design, the underlying document being often more accurate than the annotation.

Sketches and annotations are obviously also used to come up with ideas. These generations of solutions, acts of conception, are supported by drawings and elements annotations type, as well as by digital sketches. These new ideas can be directly assessed visually and graphically by the group members and teachers.

Annotations sometimes meet several of these functions, and it is not easy to establish a strict correspondence between the form of annotation and its function. Note that all these functions have been identified in all groups.

In the groups, we observe that usually, each group member uses a specific color to annotate the document. This may allow the group to identify the annotations' authors and to trace the argumentation process. But this behavior is not systematic. Participants change pen color when they have to make several sketches or annotation, not when they just have to make a quick note.

\subsection{Two distinct annotation practices}

A striking element emerging from our observation is the differentiation between two attitudes regarding the annotation practices. In particular, these attitudes concern the link between annotations and underlying documents.

The first approach is to perform many annotations in a very spontaneous way, directly on the drawings (no matter who is the author) without erasing them. It seems that for some individuals the annotation is by nature ephemeral, and therefore it can be used flexibly and intensely. This is using the environment as a temporary workspace, allowing all compositions, simulations and graphic gestures.

In the second approach, the annotations appear to have an informative and durable role. This is using the environment as a document editor. The authorship of the document is a important matter: users an- notate much less the documents created by the others, use the eraser and make drawings more accurate. From this perspective, users 'respect' documents and draw on it only to add relevant information

Both of these attitudes depend on the group in which individuals are engaged, personal preferences, and the author. When they annotate their own documents, students are more likely to use a workspace attitude: they do not hesitate to annotate their documents quickly and intensely.

\subsection{Comparison of the annotation practices between the group}

In general, the group 1 uses graphical modality for presentation: the documents are brought into the workspace by the different designers, each in turn explains the contents of the documents, using the annotation as a medium of presentation. Designers highlight essential parts of the design, add pieces of information to explain the document or draw pointing annotation to identify the elements of design to which they refer. There are few additions of information by the other students. Sets of questions and answers regularly take place between students and teachers or between students themselves and the graphical modality is used to identify the elements of design on which the discussion focuses. Most of the time in this group, the documents are only annotated by their authors and the teachers.

This practice is probably due to the operating mode of the group. As mentioned above, group 1 is characterized by a fairly decentralized design management. Thus, most of the design work is done individually, and group sessions are used to coordinate the actions of each. Those synchronous sessions are mainly used by students to communicate the choices made individually and to have them validated by the group and teachers. The annotations are used primarily for the purpose of communication and for building a shared vision of the project. The generation of solutions therefore takes place outside the meeting sessions, and synchronous DCDS sessions are used to evaluate those solutions and to reframe the problem. Students in this group use the environment mainly for editing documents.

Group 2 is characterized by a very different mode of operation. Very quickly, the group chooses a unique concept for the project and the meetings on the DCDS are used to solve important issues, to pro- 
pose ideas and make strategic decisions. Here, the annotations behaviors are directed towards the design: lots of new information is brought into the workspace, with "drawing" annotations and digital sketches. The students spend a much less time to present to each other documents, in favor of the generation of solutions and collective decision-making. The virtual sheet is considered as a shared virtual simulation space. The various documents are annotated by all group members, which indicates a real sharing of representations. All members of this group adopt workspace attitude concerning the DCDS system.

The focus here is on the generation and criticism of novel ideas. The graphical modality is less used as a tool for annotation of existing documents, than as an instrument for designing. The collaborative meetings are ideal places for solutions generation and partial evaluations. The precise resolution of the generated ideas (i.e. increasing accuracy, reducing ambiguity, choosing one solution among the several possible solutions) is done asynchronously.

Group 3 is also characterized by a different organization. Annotations are almost exclusively done by the authors of the documents, in order to convince their partners. As in Group 1, Group 3 students present themselves each other's work, but there is little interaction within the group. Most of the discussions are held between the student presenting his own work and the teachers. These discussions are sometimes punctuated by questions and answers from other participants. Here, the aim of the synchronous sessions is neither to generate all solutions, nor to collectively evaluate the propositions, but rather to convince the partners. The digital modality is essentially used to support the presentation of documents, which explains the prevalence of pointing annotations. Errors are poorly detected and poorly recovered: in-depth analysis of the proposals rarely occurs. This probably explains the lower results obtained by the group.

This functioning generates or is generated by a climate of conflict within the group. It is accompanied by an inability to agree on a unique concept or a common vision of the project. Thus, ambiguities and disagreements about the project persist until the end of the workshop. The synchronous collaboration sessions, the large number of emails exchanged between sessions, and even the intervention of teachers, fail to resolve the conflict.

\section{Role of annotation in collaborative practice}

Our results show that graphical modality is a flexible tool for collaborative design, which can serve three main purposes.

Support the communication. This is primarily to support the speech and the argumentation, by highlighting the spatial elements to which the designer refers when he explains the documents. It is also a mean to convey gestures remotely, weakly supported in our environment remotely. The graphical modality and digital sketching provides a "cheap" and quick way to perform temporary pointing gestures.

Support the design (idea generation, questions and answers). The graphical modality allows to express ideas, to complete plans and models, to ask and answer questions, to share reflections by expressing them geometrically, etc. One can thus observe sequences of questions and answers with pointing, verbal criticism with drawn counter-proposals, etc. The graphical modality therefore supports the argumentative episodes, typically observed in co-presence activities.

Support the construction of a common ground. By adding information not explicitly present on the documents, by adding elements of context and by graphically comparing several documents, the group ensure that everyone understands the same way the architectural object being designed, and share a common vision of the project.

\section{Conclusion}

This study aims at understanding the role of the graphical modality in remote collaboration in design. We followed three groups of students during a 3months workshop and observed their annotations practices during weekly synchronous distant collaborative sessions. These meetings are supported by the DCDS, a multimodal collaborative environment, which allow to communicate by speech, to see each other, and to draw and annotate on a shared space thanks to an electronic pen.

To our first question, namely "Does the graphical modality supplies people with means to manage a coupled collaboration ?", our answer is positive. The digital drawings and annotations allows some group to set up very "strong" versions of collaboration, in which each member share ideas and resources, and in which solutions are collectively elaborated and evaluated. But the system do not automatically im- 
plies that kind of methods. Rather, our observation show a flexible use of the digital drawings and annotation, which respond to our second question : "How the graphical modality is used to support the collaborative design process?". It is used to support communication, design and the construction of a common ground, which are three essential processes in collaborative design.

To enhance our system, and in general to design sketch-based communication tools, we propose two main recommendations.

- Support communication and design activities by specific means. The idea is to reconcile the two attitudes: "document edition" (which necessitate lasting annotations) and "workspace" (in whom a lot of annotations are temporary). Using gesture recognition or temporary traces tools may be an efficient solution.

- Support seamless integration with other tools. The vast majority of collaborative activity takes place on previously made document. The system should favor an easy import and export of the documents, to enhance possibilities and allow more flexible organization.

\section{Aknowledgements}

This work is funded by a grant from the Research Council of the University of Liège, and by the ARC grant, financed by the French Community of Belgium. The authors wish to thank all the students who participated to the study.

\section{References}

[1] Burkhardt, J-M., Détienne, F., Hebert, A.-M.., Perron, (2009) Assessing the "Quality of Collaboration" in Technology- Mediated Design Situations with Several Dimensions. Proceedings of INTERACT 2009, , pp. 157-160

[2] Burkhardt, J-M., Détienne, F., Hebert, A.-M.., Perron, L. , Safin, S. Leclercq, P. (2009) An approach to assess the quality of collaboration in technology-mediated design situations Proceedings of ECCE 2009 : European Conference on Cognitive Ergonomics. Helsinki, Septembre

[3] Carroll, J. M., Neale, D. C., Isenhour, P. L., Rosson, M. B., \& McCrickard, D. S. (2003). Notification and awareness : synchronizing task-oriented collaborative activity. International Journal Of Human-Computer Studies, 58, 605-632.

[4] Clarck, H., \& Brennan, S. (1991). Grounding in communication. In L. Resnick, J. Levine \& S. Teasley (Eds.), Perspectives on Socially Shared Cognition. Washington: American Psychological Association.
[5] Darses, F. (2004). Processus psychologiques de résolution collective des problèmes de conception : contribution de la psychologie ergonomique. Unpublished HDR - habilitation à Diriger des Recherches, Université Paris V - René Descartes.

[6] Darses, F., Falzon, P., \& Béguin, P. (1996). Collective design processes. . Paper presented at the COOP 96, Second International Conference on the Design of Cooperative Systems, Juan-les- Pins.

[7] Détienne, F., Boujut, J.-F., \& Hohmann, B. (2004). Characterization of collaborative design and interaction management activities in a distant engineering design situation. Paper presented at the COOP 2004 - Cooperative systems design: scenario-based design of collaborative systems.

[8] Elsen C., Leclercq P., 2008. A sketching tool to support collaborative design. CDVE'08, 5th International Conference on Cooperative Design, Vizualisation and Engineering, Mallorca, Spain.

[9] Kubicki S., Bignon J-C., Lotz J., Gilles Halin G., Elsen C. \& Leclercq P., 2008. Digital Cooperative Studio. ICE 2008 14th International Conference on Concurrent Enterprising, Special session ICT-supported Cooperative Design in Education, Lisboa, Espagne.

[10]Roschelle, J., \& Teasley, S. (1994). The construction of shared knowledge in collaborative problem solving. In C. E. O'Malley (Ed.), Computer Supported Collaborative Learning (pp. 69-97). Heidelberg: Springer-Verlag.

[11] Safin, S., Delfosse, V., \& Leclercq, P. (2010) Mixed-reality prototypes to support early creative design. In E. Dubois, P. Gray \& L. Nigay (Eds). The Engineering of Mixed Reality Systems. London : Springer

[12] Safin, S., Juchmes, R. \& Leclercq, P. (in press) Du crayon au stylo numérique : influences des IHM à stylo et des interprétations numériques sur l'activité graphique en tâches de conception. Journal d'Interaction Personne-Système.

[13] Safin, S. \& Leclercq, P. (2009). User studies of a sketch-based collaborative distant design solution in industrial context. Proceedings of CDVE 2009. The 6th International Conference on Cooperative Design, Visualization and Engineering. Luxembourg, Septembre.

[14] Safin, S., Verschuere, A., Defays, A., Burkhardt, J-M. \& Détienne, F. (2010) Quality of collaboration in a distant collaborative architectural educational setting. Workshop W1: Analysing the quality of collaboration in task-oriented computermediated interactions, in COOP 2010 : 9th International Conference on the Design of Cooperative Systems. Aix-enProvence, May 2010

[15] Spada, H., Meier, A., Rummel, N., \& Hauser, S. (2005). A new method to assess the quality of collaborative process in CSCL. Paper presented at the Conference on Computer support for collaborative learning : learning 2005: the next 10 years!

[16] Stempfle, J., \& Badke-Schaub, P. (2002). Thinking in design teams-an analysis of team communication. Design studies, 23, 473-496.

[17] Visser, W. (2001). Conception individuelle et collective. Approche de l'ergonomie cognitive. Le Chesnay, France: INRIA Institut national de la Recherche en Informatique et Automatique. 